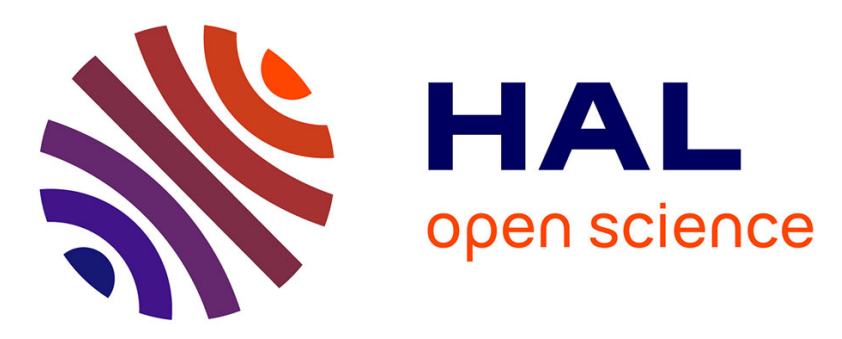

\title{
Testing Oxygen Nuclear Data Libraries in Research Center Rez
}

Martin Schulc, Michal Kostal, Ezen Vnovak, Jan Simon, Luiz Leal

\section{To cite this version:}

Martin Schulc, Michal Kostal, Ezen Vnovak, Jan Simon, Luiz Leal. Testing Oxygen Nuclear Data Libraries in Research Center Rez. Journal of Nuclear Engineering and Radiation Science, 2020, 7 (2), pp.021501. 10.1115/1.4048763 . hal-03208868

\section{HAL Id: hal-03208868 \\ https://hal.science/hal-03208868}

Submitted on 26 Apr 2021

HAL is a multi-disciplinary open access archive for the deposit and dissemination of scientific research documents, whether they are published or not. The documents may come from teaching and research institutions in France or abroad, or from public or private research centers.
L'archive ouverte pluridisciplinaire HAL, est destinée au dépôt et à la diffusion de documents scientifiques de niveau recherche, publiés ou non, émanant des établissements d'enseignement et de recherche français ou étrangers, des laboratoires publics ou privés.

\section{(c)(1)}

Distributed under a Creative Commons Attribution| 4.0 International License 


\title{
TESTING OXYGEN NUCLEAR DATA LIBRARIES IN RESEARCH CENTRE REZ
}

\author{
Martin Schulc, Michal Košt’ál, Evžen Novák and Jan Šimon \\ Research Centre Rez Ltd \\ Husinec-Řež 130, 250 68, Czech Republic \\ martin.schulc@cvrez.cz; michal.kostal@cvrez.cz; evzen.novak@cvrez.cz; \\ jan.simon@cvrez.cz \\ Luiz Leal \\ Institut de Radioprotection et de Surete Nucleaire \\ 31 Avenue de la Division Leclerc, 92260, Fontenay-aux-Roses, France \\ luiz.1eal@irsn.fr
}

\begin{abstract}
Oxygen belongs to the group of the most important isotopes in the nuclear data field. The aim of this paper is to validate various oxygen nuclear data libraries in different scenarios with high content of oxygen. For this purpose, fast neutron spectra were measured by a stilbene scintillation detector in the region of $1-10 \mathrm{MeV}$ in the three model cases involving two ${ }^{252} \mathrm{Cf}$ neutron source experiments and light water reactor. The cases include measurements of leakage spectra using ${ }^{252} \mathrm{Cf}$ neutron source placed in the centres of the light water and heavy water spheres of $30 \mathrm{~cm}$ diameter. Following measurements were carried out inside the concrete biological shielding of the VVER-1000 mock-up simulator in the LR-0 reactor and in the dry channel located in the centre of the special core placed in the LR-0 reactor. In the case of the special core, symmetric active core consisted of six standard fuel assemblies which surround the experimental dry module, where the fast neutron spectrum was measured. The measured neutron spectra were compared with MCNP6 transport code calculations in ENDF/B-VII.1, ENDF/B-VIII.0., JENDL-4.0 and IRSN ${ }^{16} \mathrm{O}$ nuclear data evaluations. Experimental results for all cases follow a similar trend. All considered libraries underestimate experimental measurement in the region of 3-4 MeV in all experimental cases.
\end{abstract}

Key Words: LR-0 reactor, ${ }^{252} \mathrm{Cf}$, cross section, validation, ${ }^{16} \mathrm{O}$

\section{Introduction}

Oxygen is one of the most important isotopes in the nuclear data field. Numerous nuclear data applications require an excellent knowledge of oxygen cross-sections. As oxygen is abundant in concrete, light water, heavy water and nuclear fuel, the accurate knowledge of cross-section data and related uncertainties is crucial for the nuclear reactor analysis and design and nuclear criticality safety as well as for the processing and disposal of nuclear waste. Cross section for ${ }^{16} \mathrm{O}(\mathrm{n}, \alpha)^{13} \mathrm{C}$ reaction affects the reactivity of light water reactors and is responsible for the production of helium in fuel pins and claddings and is therefore important for the behavior of these parts.

The set of experiments was performed to validate oxygen evaluation for ENDF/B-VII.1 [1], ENDF/B-VIII.0 [2], JENDL-4.0 [3] and IRSN [4] nuclear data libraries in three different scenarios situated into a high oxygen content environment. Measured spectra were compared with MCNP6.1 [5] code calculations using aforementioned libraries. All calculations were performed at the room temperature. The influence of the nuclear data libraries onto nuclear transport problem was investigated by changing of only ${ }^{16} \mathrm{O} \mathrm{XS}$ ACE file. The other ACE files employed were taken from ENDF/B-VII.0 library [6]. All 
cross section files were provided as ACE files. In the case of the water spheres, the input ${ }^{252} \mathrm{Cf}$ spontaneous neutron fission spectrum was taken from the IRDFF webpage [7]. Fast neutron spectra were measured using stilbene scintillation detector with time pulse shape discrimination in all experiments. The first part of experiments concerns heavy water and light water spherical benchmarks with ${ }^{252} \mathrm{Cf}$ source. The second part involves light water reactor.

\section{Experiment evaluation}

The performed experiments consist of three different parts:

\subsection{Light and Heavy water spheres}

The ${ }^{252} \mathrm{Cf}$ neutron source was placed in the centre of a heavy or light water sphere using a pneumatic flexo-rabbit system. Heavy or light water sphere has a diameter of $d=29.997$ $\mathrm{cm}$ and is covered with a steel coating of thickness $t=0.078 \mathrm{~cm}$ and in the case of heavy water sphere contains 99.36 wt. \% of heavy water, the rest is a light water [8]. The spheres were hanged up to a distance of 2 metres from the floor to minimize the room effect. Fast neutron leakage spectra were measured $100 \mathrm{~cm}$ from the centre of the water sphere using stilbene detector [9]. The geometry of the experimental arrangement can be found in the Figure 1. More details concerning the experiment can be found in [10].

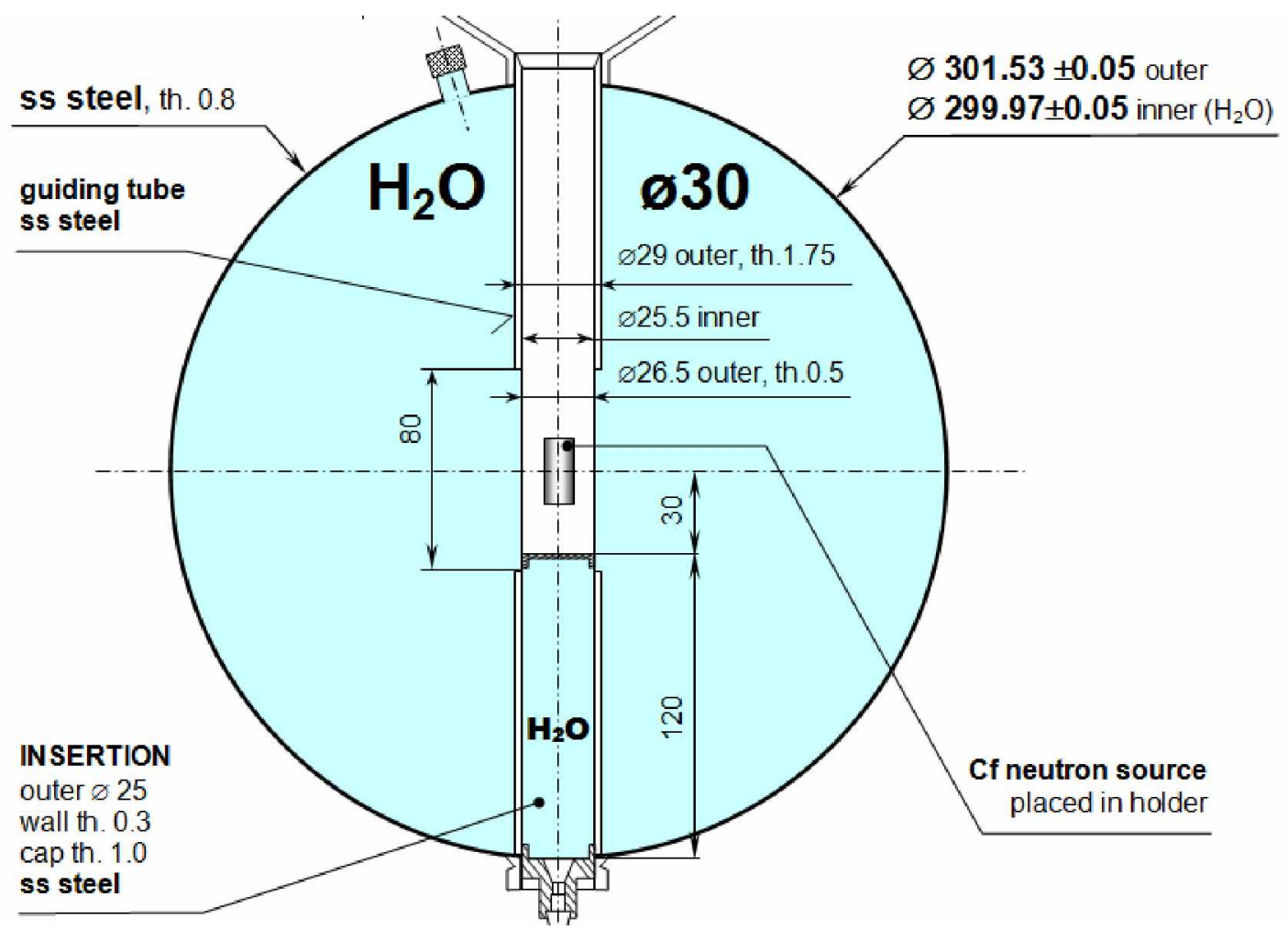

Figure 1: Light water sphere design with neutron source. Heavy water sphere has same dimensions. All dimensions shown are in $\mathrm{mm}$.

\subsection{Special Core}

The light water pool type zero-power reactor LR-0 has standard fuel assemblies which are identical to the VVER-1000 fuel assemblies in a radial direction but they are shorter in the axial direction. It can operate continuously at maximum power of $1 \mathrm{~kW}$. The LR-0 
reactor is flexible in the core-supporting structures which allow the use of the fuel assemblies of different types and enrichment even with variable lattice pitch. The length of fissile column is $125 \mathrm{~cm}$. Reactor power monitoring in reactor experiments was carried out by an independent measurement using a compensated boron chamber placed in the lateral moderator region of the reactor core. The discrepancy between proportionality of the monitors was estimated independently by means of measurement with a well-defined ${ }^{3} \mathrm{He}$ detector [11].

The source strength is expressed by means of a scaling factor K. It is a number which is needed to multiply the MCNP6 results to obtain the calculation results per unit response of the neutron monitor. Its physical meaning is the core neutron emission rate. This factor is determined by means of comparison between calculated and measured reaction rates of a gold and nickel activation detectors, see [12].

Active core consisted of six standard fuel assemblies with the approximately $3.3 \mathrm{wt} \%$ enrichment which surround the experimental dry module, where the fast neutron spectrum was measured, see Figure 2.

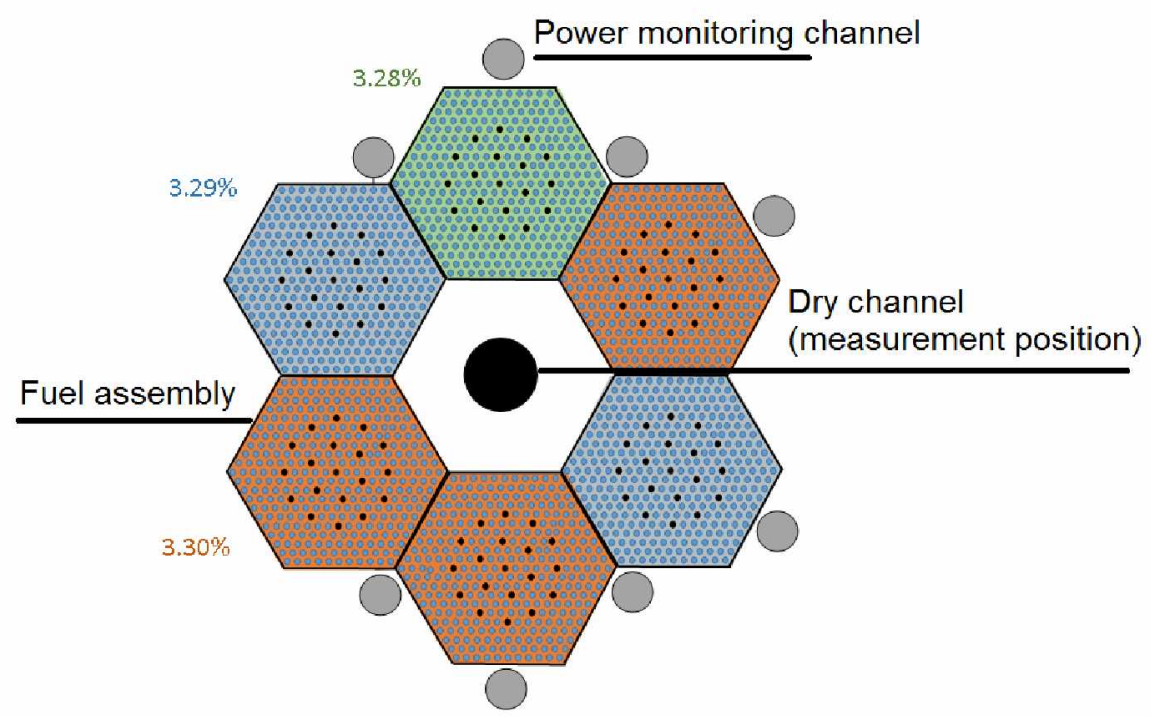

Figure 2: Special core placed in the LR-0 reactor.

\subsection{Biological shielding of the reactor}

Fast neutron spectra in the model of VVER-1000 concrete biological shielding, located behind LR-0 reactor vessel, were realized with the VVER-1000 Mock-Up core [13] as neutron source. VVER-1000 Mock-Up is a radial, full scale VVER-1000 model established for reactor dosimetry purposes. The Mock-Up core consists of 32 dismountable VVER-1000 fuel assemblies with enrichment $2.0 \mathrm{wt} \%, 3.0 \mathrm{wt}$. \% and 3.3 wt. $\%$ of ${ }^{235} \mathrm{U}$. It is placed inside an aluminium cylindrical tank $1.6 \mathrm{~cm}$ thick with inner diameter of $350 \mathrm{~cm}$. Criticality is adjusted using two control clusters with three absorbing $\mathrm{B}_{4} \mathrm{C}$ rods, exposed to $60.4 \mathrm{~cm}$ above the bottom of the fissile column. The active core is fully flooded to $25 \mathrm{~cm}$ above the upper end of the fuel. Demineralized water with diluted boric acid of concentration $4.68 \mathrm{~g} / \mathrm{l}$ is used as a moderator. The radial cross-section 
scheme with measurement point inside the biological shielding concrete is shown in Figure 3 .

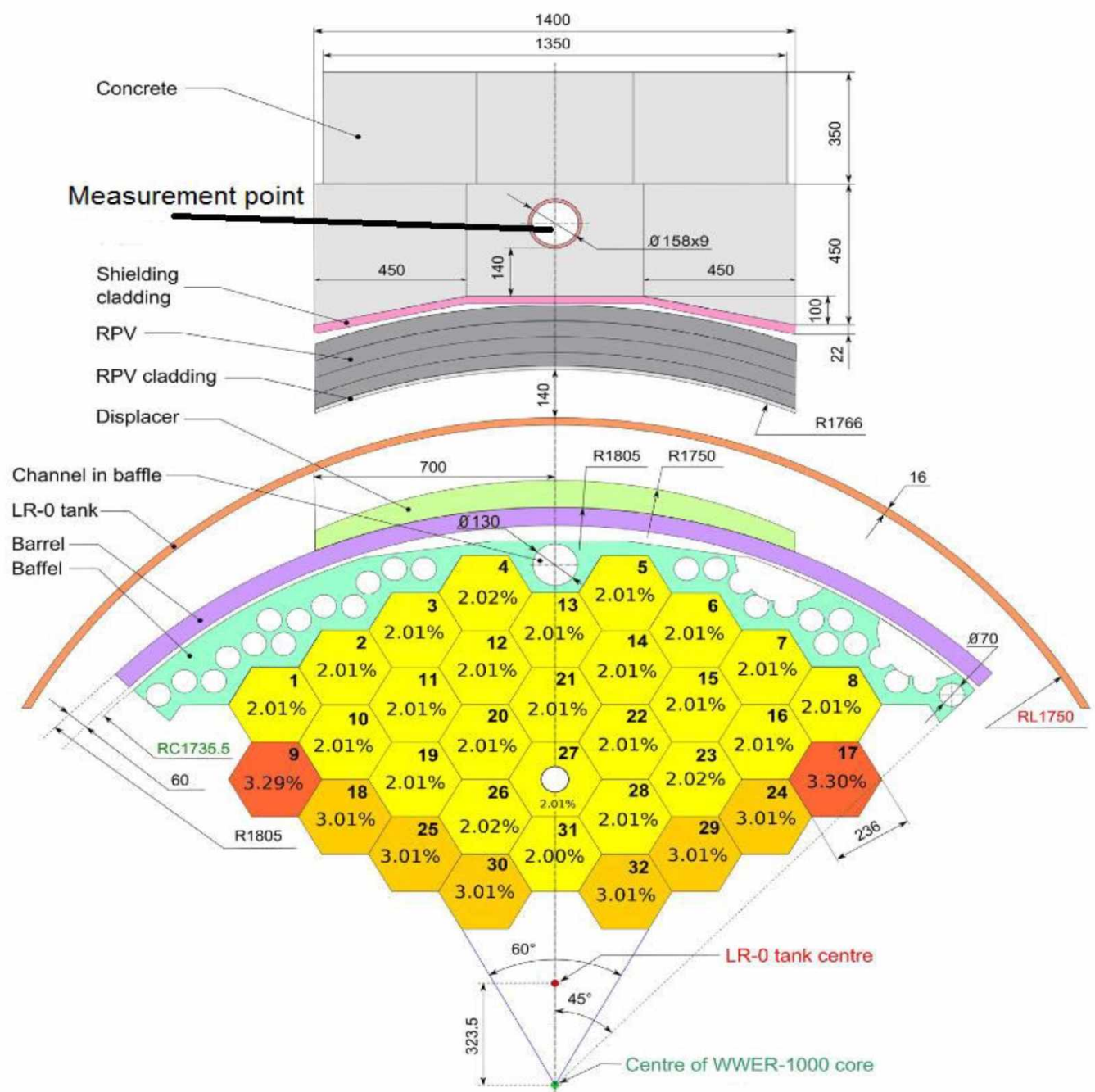

Figure 3: Radial section of VVER-1000 transport benchmark at LR-0 reactor. (Measurement point is highlighted.)

\subsection{Fast neutron flux measurement}

Fast neutron spectra were measured by the proton recoil method using a stilbene scintillation detector with a fully digitized two-parameter spectrometric system in the interval from 1.0 to $10.0 \mathrm{MeV}$ in groups with step of $100 \mathrm{keV}$, for technical details see [14]. The size of the cylindrical stilbene scintillator used for the measurements was always $10 \mathrm{~mm} \times 10 \mathrm{~mm}$. The neutron spectra are evaluated from the measured proton recoil spectra by means of deconvolution using Maximum Likelihood Estimation, see [15]. Used stilbene crystal was tested in quasi monoenergetic neutron beam behind $\mathrm{Si}$ filter [16].

The efficiency calibration employs a measurement using a pure ${ }^{252} \mathrm{Cf}$ neutron source. The substantial sources of uncertainty in the measurement were: an energy calibration uncertainty of $2.5-5.5 \%$, an uncertainty in the efficiency crystal calibration factor $2.2 \%$, and an uncertainty in the total emission of the neutron ${ }^{252} \mathrm{Cf}$ source $1.3 \%$. Total 
measurement uncertainty, including statistical uncertainty and dispersion between measurements, is between approximately $2.8 \%-16.1 \%$ in the measured region, with highest uncertainties for intervals being 1-1.4 MeV and 9-10 MeV. Generally, light water environment measurement has higher uncertainty due to the higher gamma background.

\section{Results}

Concerning the water spheres results, Figure 4 shows the $\mathrm{C} / \mathrm{E}$ (Calculation to Experiment) ratios for the heavy water sphere including experimental uncertainties. Gaussian energy broadening function, which takes into account energy resolution of the detector, was implemented for all calculated spectra and then compared with experimental data. Overall, the agreement is best for the ENDF/B-VIII.0 and IRSN oxygen evaluation. All libraries underestimate experiment up to $14 \%$ in the region of 3-4 MeV. JENDL-4.0 library significantly overpredicts measured data in the region of 4-7 MeV.

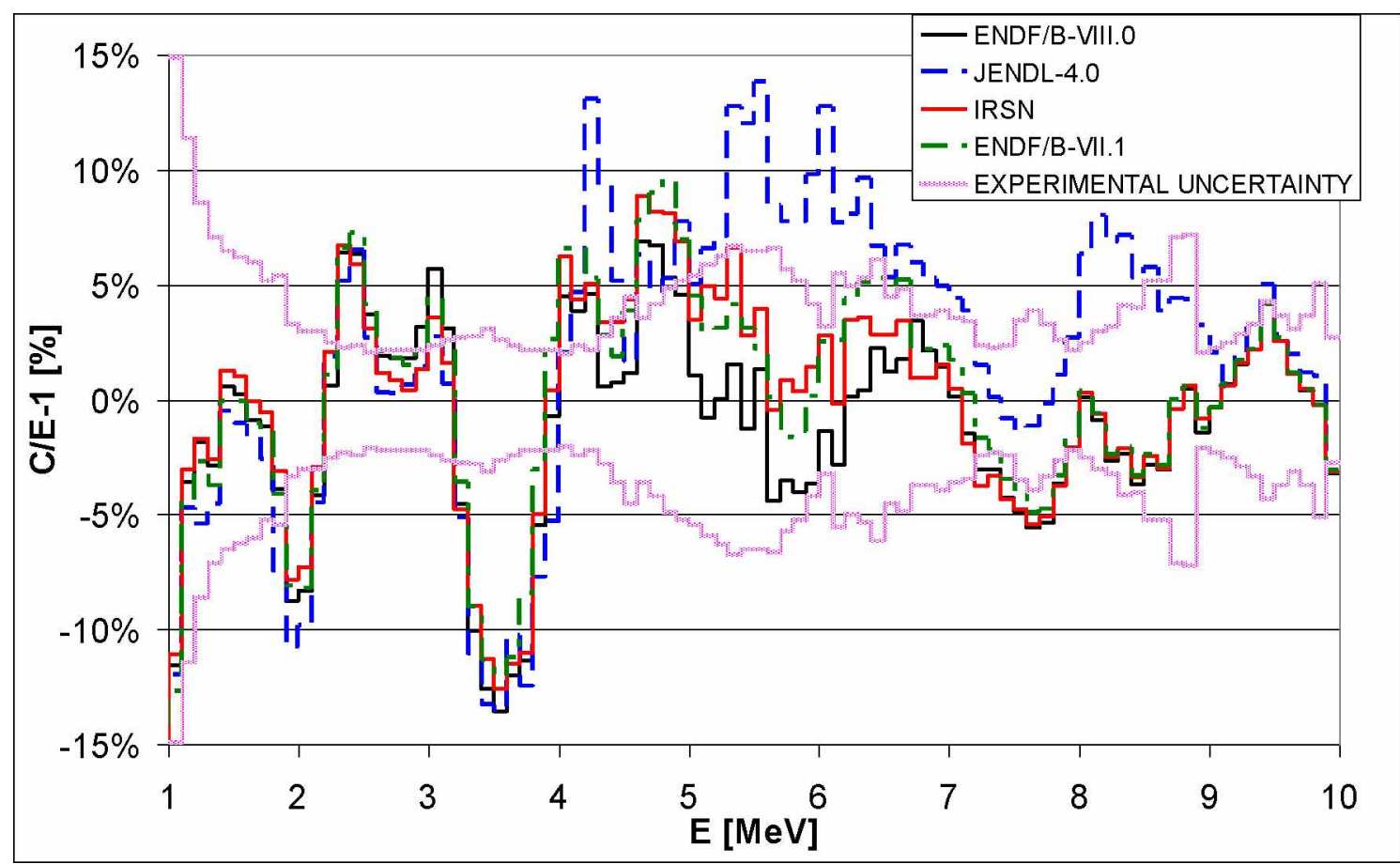

Figure 4: Heavy water neutron leakage spectra comparison via C/E-1.

Figure 5 shows the $\mathrm{C} / \mathrm{E}$ ratios with experimental uncertainties for the light water sphere. It compares various libraries calculations, where Gaussian energy broadening function was implemented. The trend for all libraries is very similar as in the heavy water case. Good agreement is achieved with all libraries under study in the region of 1-3 MeV. However, again all libraries underestimate experiment up to $15 \%$ in the region of 3-4 $\mathrm{MeV}$. For higher energies, IRSN and ENDF/B oxygen evaluations achieve good agreement in the region of 4-6.5 MeV unlike JENDL-4.0 library which overestimates experimental results. Good agreement is achieved for energies higher than $6.5 \mathrm{MeV}$ with all libraries.

Figure 6 displays results for the special core. The results are satisfacory in the whole energy range under study in all oxygen evaluations. The libraries perform similarly up to 3.5 MeV from where the results are different for different evaluations. Again, all libraries 
underestimate experiment in the range of 3-4 MeV. For higher energies, i.e. up to 9.5 $\mathrm{MeV}$ all evaluations are within uncertainties. The only region which is beyond experimental uncertainties is around $2.5 \mathrm{MeV}$ and $3.5 \mathrm{MeV}$, it holds for all evaluations.

Figure 7 shows $\mathrm{C} / \mathrm{E}$ ratios with experimental uncertainties for the measurement in the biological shielding of VVER-1000 mock-up. JENDL-4.0 oxygen evaluation highly overpredicts experiment in the energy range of 4-9 MeV. The highest discrepancies which accounts up to over $80 \%$ for this evaluation are in the energy range of 5.0-7.0 $\mathrm{MeV}$. All other evaluations also overpredict in this energy range. The best agreement is achieved using ENDF/B-VIII.0.evaluation in this energy range and also overall. Notice the consistent underestimation of all libraries in the energy range of 3-4 MeV.

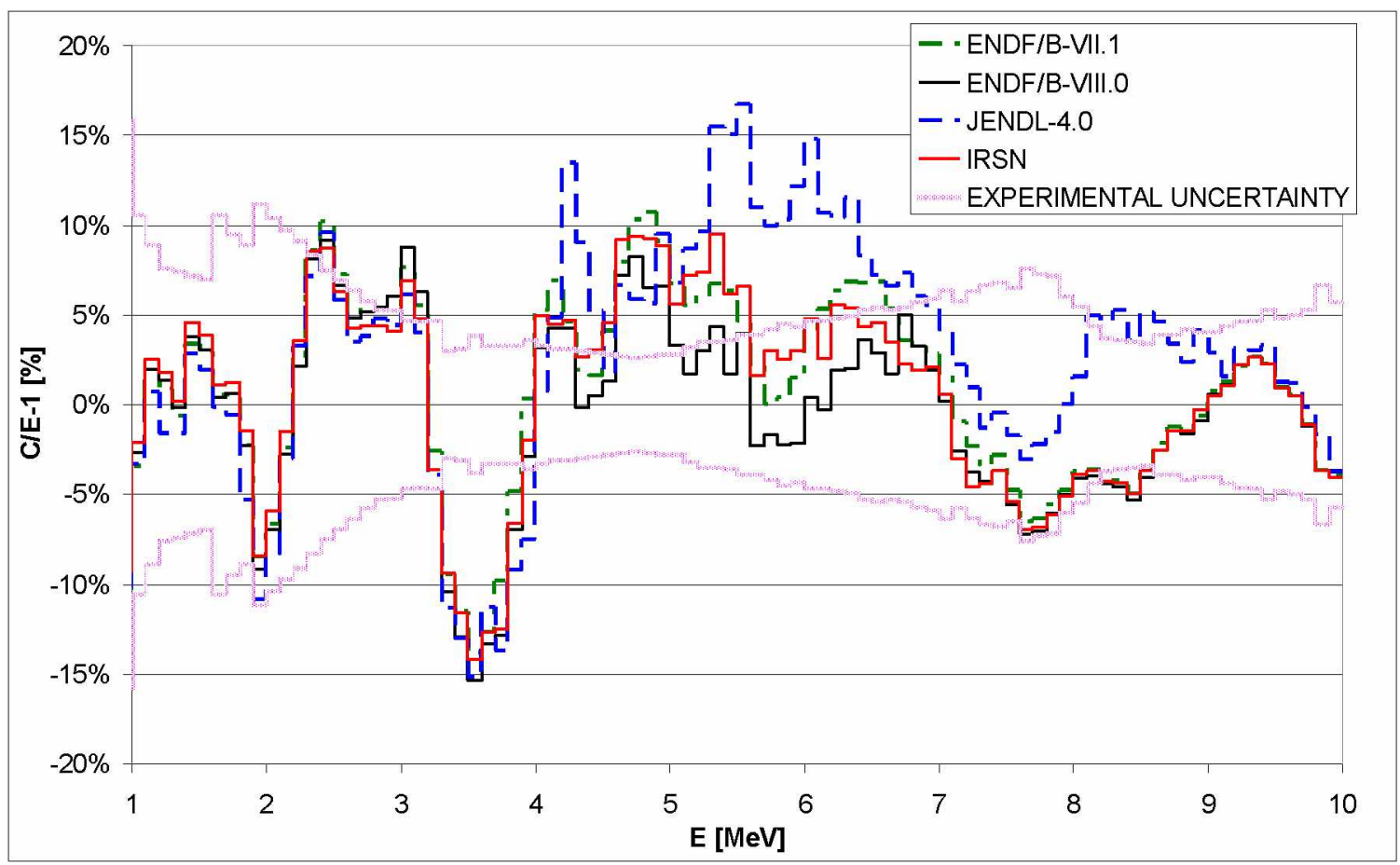

Figure 5: Light water neutron leakage spectra comparison via C/E-1. 


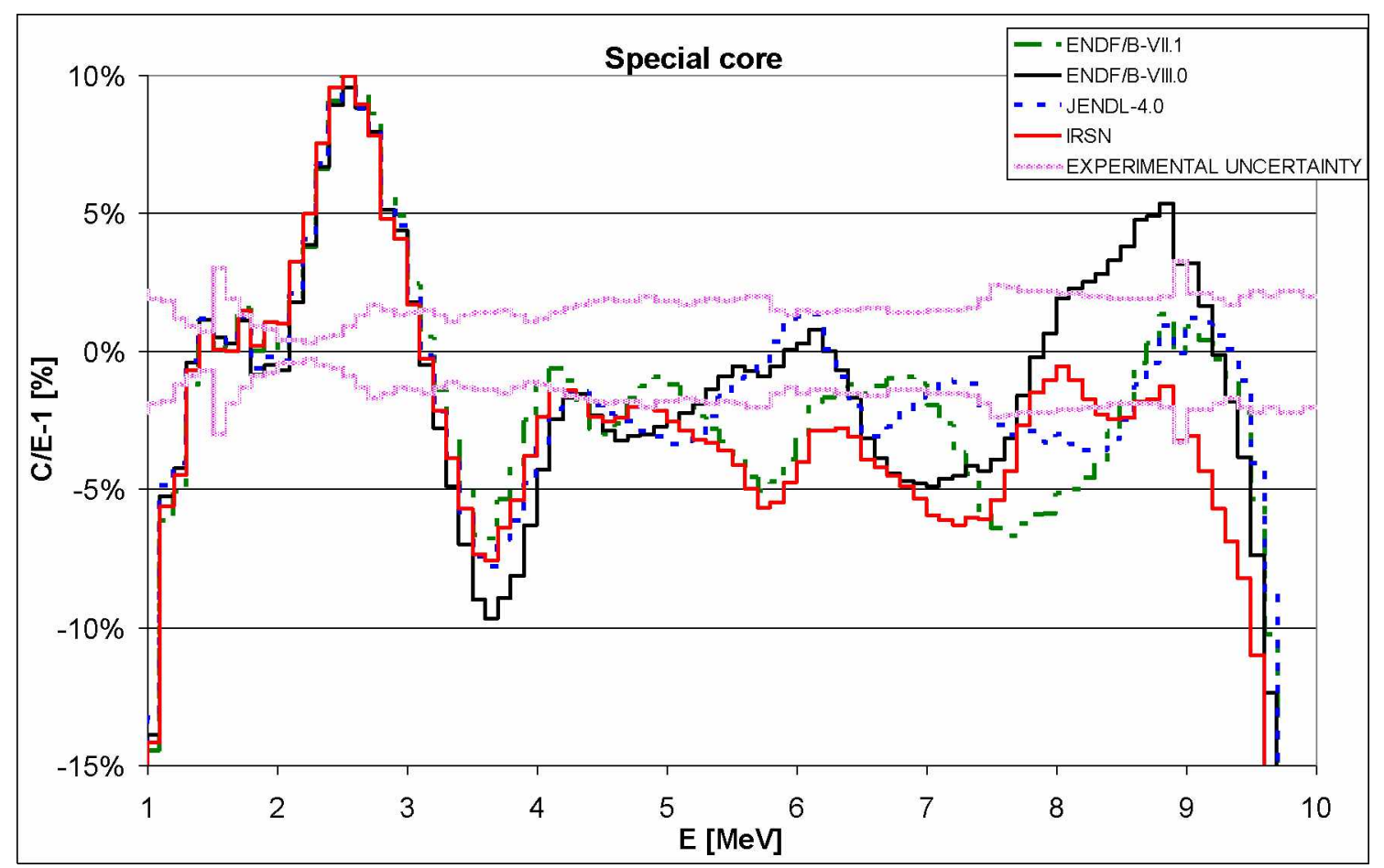

Figure 6: Special core C/E-1 comparison for different oxygen evaluations.

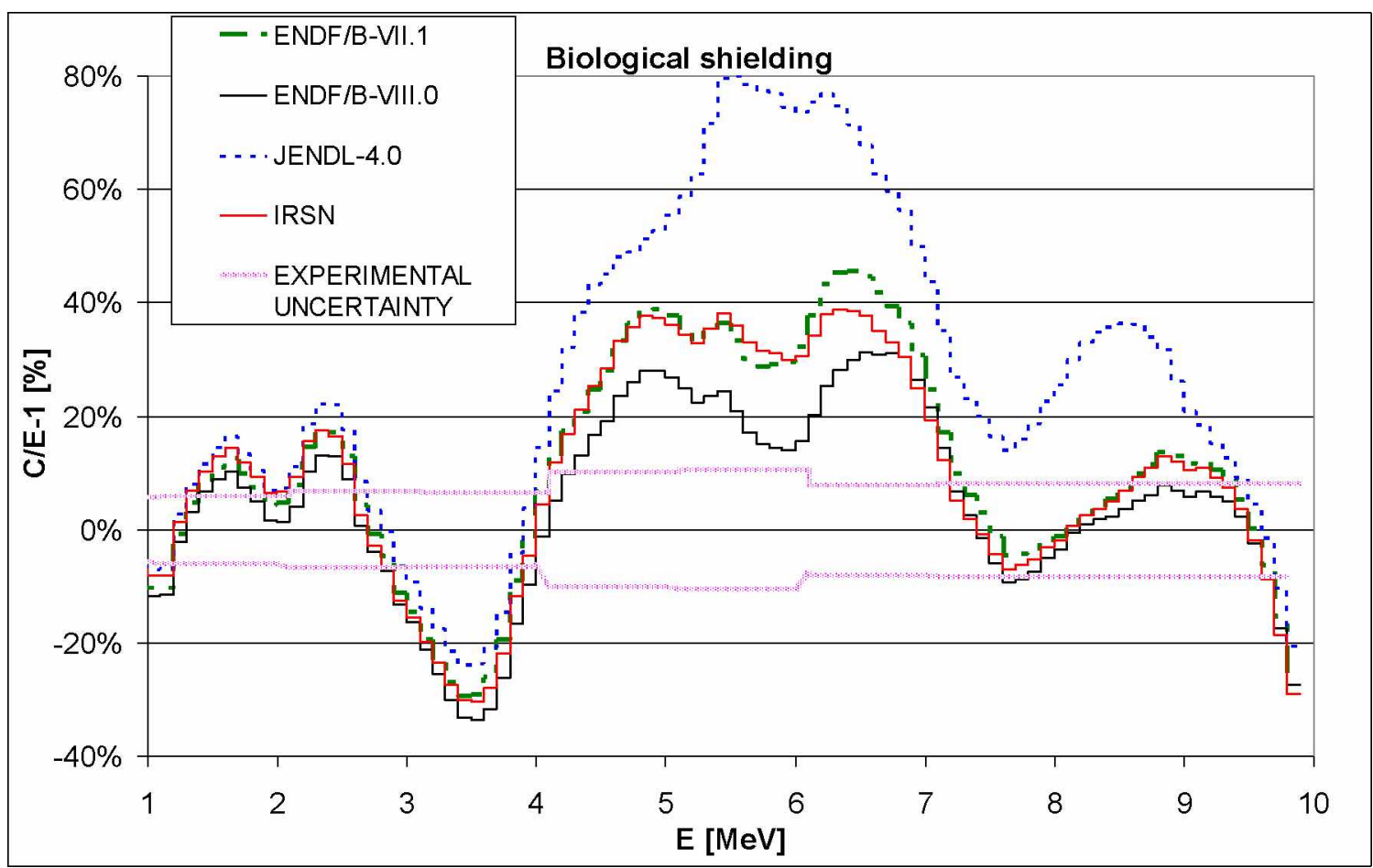

Figure 7: Biological shielding C/E-1 comparison for different oxygen evaluations.

Figure 8 shows calculation to experiment ratio (C/E-1) with experimental uncertainties in the energy range of 1-10 MeV using ENDF/B-VIII.0 library. Good agreement is achieved for all cases except the measurement in the biological shielding of LR-0 reactor. However, this is a very complex case primarily influenced by other nuclides rather than oxygen. Interesting fact is that ENDF/B-VIII.0.underestimates experimental measurement in the region of 3-4 $\mathrm{MeV}$ for all cases. 


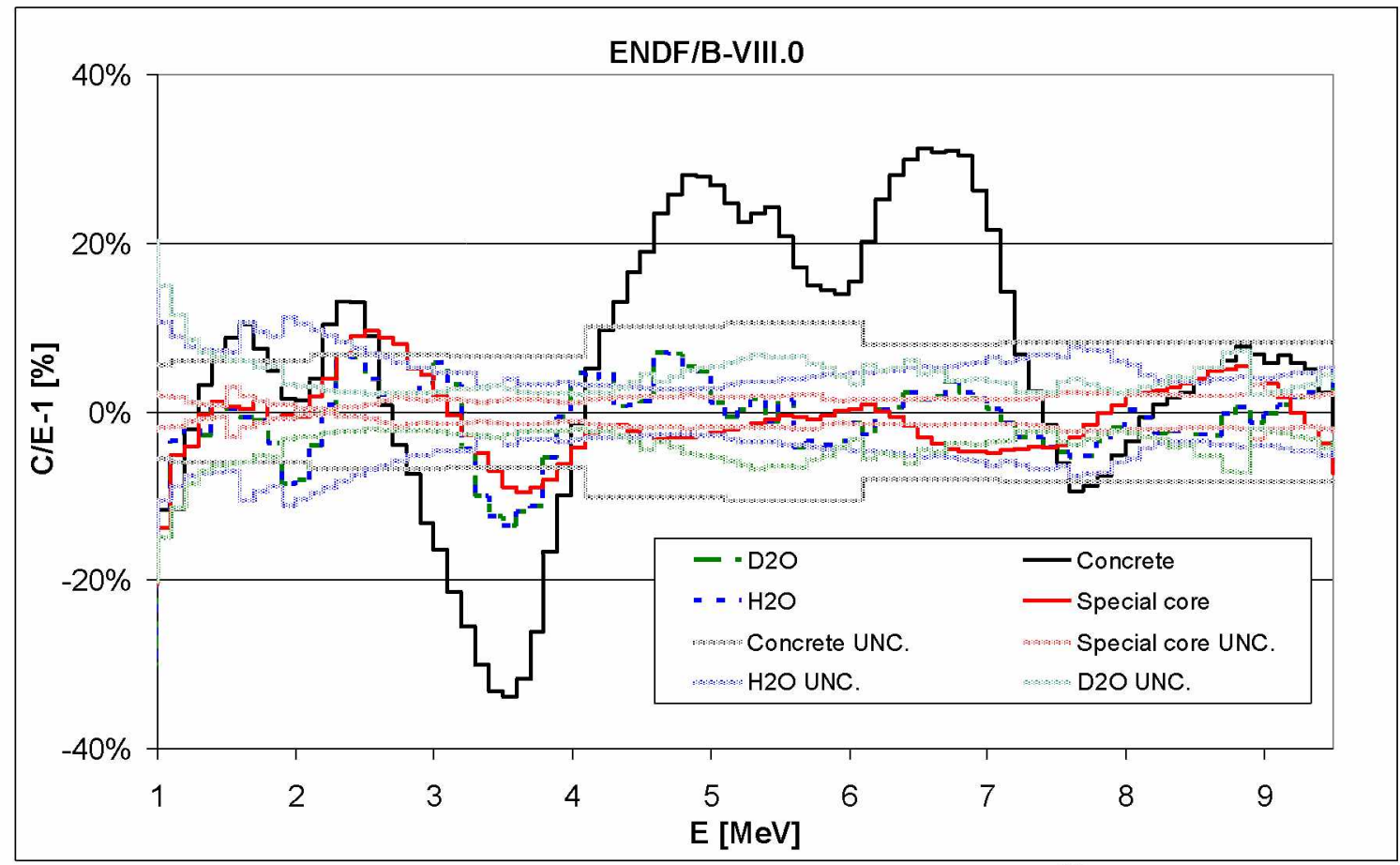

Figure 8: Calculation to experiment ratio using ENDF/B-VIII.0 ${ }^{16} \mathrm{O}$ evaluation for all mentioned experiments.

The elastic angular distribution for emitted neutrons determines the energy lost during collisions and thus affects the neutrons slowing down and their removal to below the given detection energy limit of $1 \mathrm{MeV}$. The observed neutron fluence difference between JENDL-4.0 and ENDF/B-VIII.0.libraries can be caused by this. The angular distributions differ__ significantly as it is shown in Figure 9.

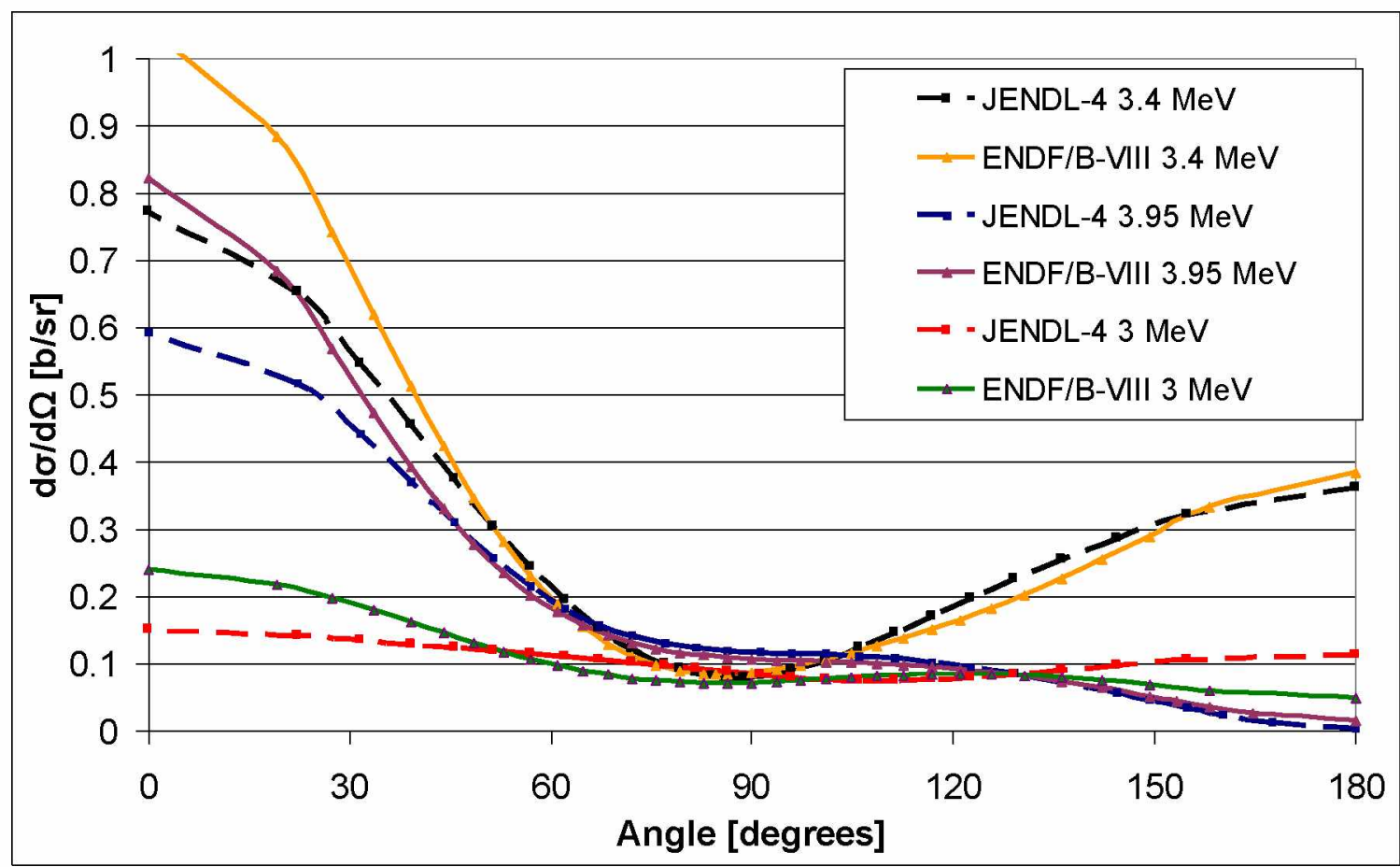

Figure 9: Elastic angular distributions in JENDL-4.0 and ENDF/B-VIII.0 libraries for energies between 3 and $4 \mathrm{MeV}$. 


\section{CONCLUSIONS}

The presented work focused on the validation of the different oxygen evaluations by means of comparison of the calculated and measured neutron spectra in the range of 1-10 $\mathrm{MeV}$.

The experimental neutron fluences for the light and heavy water spheres were compared with calculations with ENDF/B-VII.1, ENDF/B-VIII.0 and JENDL-4.0 nuclear data libraries. In the case of light water sphere good agreement is achieved with all libraries in the region of 1-3 MeV. All libraries underestimate experiment in the region of 3-4 MeV. For higher energies, ENDF/B libraries achieve agreement within experimental uncertainties unlike JENDL-4.0 library which overestimates experimental results in the region of 4-6.5 MeV. In the case of the heavy water sphere, all libraries underestimate experiment in the region of 3-4 MeV and JENDL-4.0 library overestimates experimental results in the region of $4-6.5 \mathrm{MeV}$ as it is in the case of the light water sphere.

In the case of the special core all evaluations results are within experimental uncertainties except energy regions around $2.5 \mathrm{MeV}$ and $3.5 \mathrm{MeV}$. In the energy range of 1.0-3.5 MeV all evaluations behave similarly.

The results for the biological shielding of VVER-1000 mock-up are worst from all cases under study. JENDL-4.0 oxygen evaluation gives the worst results with discrepancies over $80 \%$ for JENDL-4.0 in the energy range of $5-7 \mathrm{MeV}$. The best agreement is achieved with ENDF/B-VIII.0.library.

It is worth noting to emphasize that there is a similar underprediction in all cases in the energy range of 3-4 MeV for all evaluations and cases.

Concerning ENDF/B-VIII.0 library, there was made a large correction in ${ }^{16} \mathrm{O}(\mathrm{n}, \alpha)$ cross section in comparison to preceding versions. Furthermore, the measurements of ${ }^{16} \mathrm{O}(\mathrm{n}, \alpha)$ and ${ }^{16} \mathrm{O}(\mathrm{n}$,abs) in the energy range of $2-20 \mathrm{MeV}$ is included in NEA's high priority list. The total ${ }^{16} \mathrm{O}(\mathrm{n}, \mathrm{tot})$ cross section plays an important role in our understanding of neutron reactions in oxygen, in part because of its influence on the $(n, \alpha)$ reaction via unitarity [2]. Future experiments should focus on this issue. Figure 10 shows ${ }^{16} \mathrm{O}(\mathrm{n}, \alpha)$ cross section in various libraries together with available data from EXFOR database [17]. 


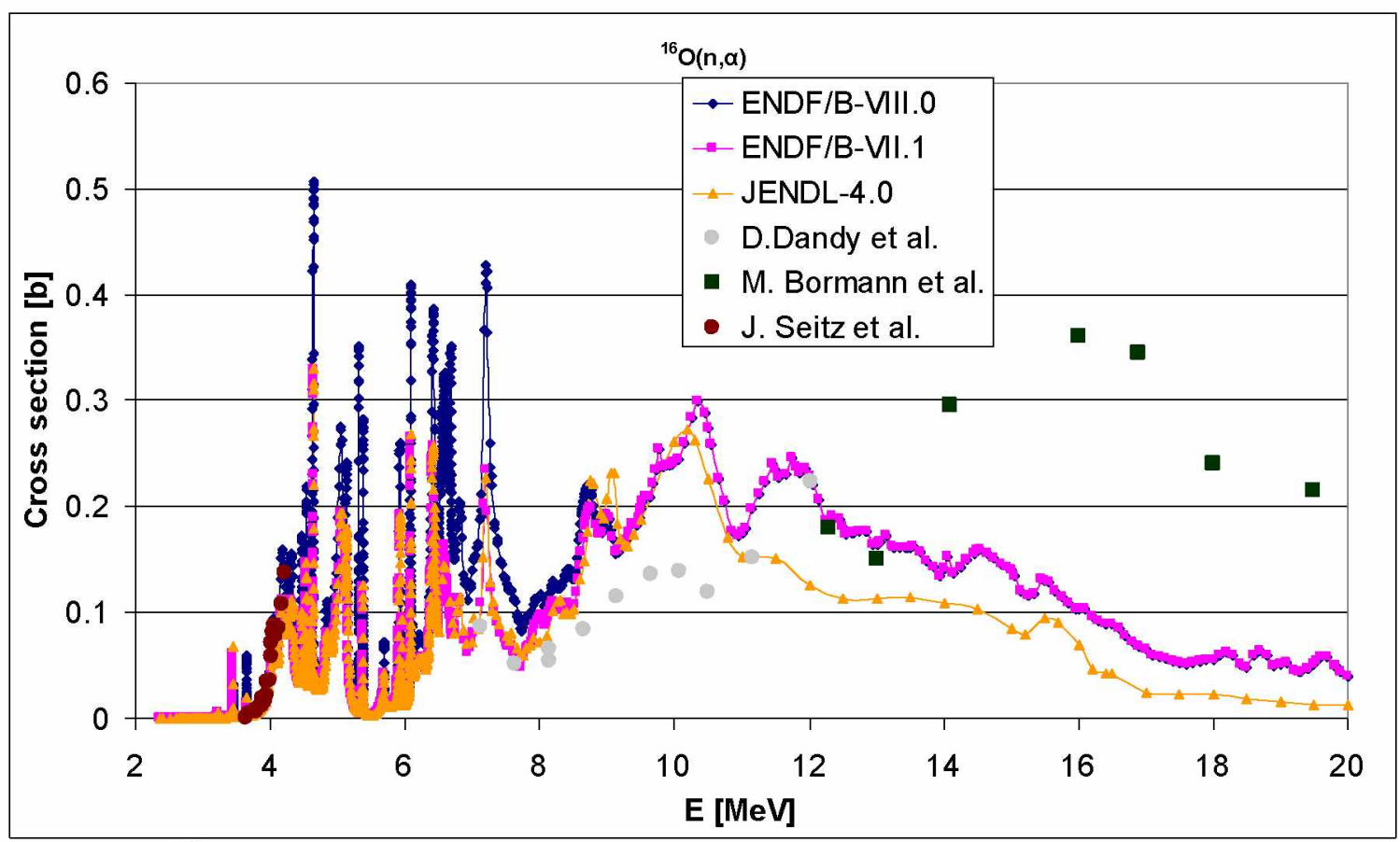

Figure $10:{ }^{16} \mathrm{O}(\mathrm{n}, \alpha)$ cross section in various libraries.

\section{ACKNOWLEDGMENTS}

Presented results were obtained with the use of infrastructure Reactors LVR-15 and LR-0, which is financially supported by the Ministry of Education, Youth and Sports - project LM2015074 and also financially supported by the Ministry of Education, Youth and Sport Czech Republic - project LQ1603 Research for SUSEN. This work has been realized within the SUSEN Project (established in the framework of the European Regional Development Fund (ERDF) in project CZ.1.05/2.1.00/03.0108 and of the European Structural Funds and Investment Funds (ESIF) in the project CZ.02.1.01/0.0/0.0/15_008/0000293), which is financially supported by the Ministry of Education, Youth and Sports - project LM2015093 Infrastructure SUSEN.

\section{REFERENCES}

1. M.B. Chadwick, P. Obložinský et al., "ENDF/B-VII.1: next generation evaluated nuclear data library for nuclear science and technology", Nucl. Data Sheets 112, pp. 2887, (2011).

2. D.A. Brown, M.B. Chadwick, R. Capote et al, "ENDF/B-VIII.0: The 8th Major Release of the Nuclear Reaction Data Library with CIELO-project Cross Sections, New Standards and Thermal Scattering Data", Nucl. Data Sheets, 148 (2018), pp. 1142.

3. K. Shibata, O. Iwamoto, T. Nakagawa, N. Iwamoto et al., "JENDL-4.0: A new library for nuclear science and engineering“" J. Nucl. Sci. Technol., 48, pp. 1-30, (2011).

4. L. Leal, E. Ivanov et al. "Resonance Parameter and Covariance Evaluation for ${ }^{16} \mathrm{O}$ up

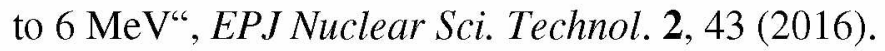

5. T. Goorley et al. "Initial MCNP6 Release Overview", Nuclear Technology, 180, pp 298-315 (2012). 
6. M. B. Chadwick, P. Obložinský, M. Herman et al, "ENDF/B-VII.0: Next Generation Evaluated Nuclear Data Library for Nuclear Science and Technology", Nuclear Data Sheets, Volume 107, pp. 2931-3060, (2006).

7. IRDFF: the IAEA Coordinated Research Projecton Testing and Improving the International Reactor Dosimetry and Fusion File (IRDFF),_https://wwwnds.iaea.org/IRDFFtest, (2016).

8. B. Jánský, P. Otopal and J. Brabec, "The production and filling of $\mathrm{D}_{2} \mathrm{O}$ sphere for neutron metrology use", (in Czech), report NRI Rez No 9755 R,D, (1992).

9. V.I. Kuchtevic, O.A. Trykov, L.A. Trykov, Odnokrystalnyj scintilljacionnyj spektrometr, Atomizdat, Moscow (1971), (in Russian).

10. M. Schulc, M. Košt'ál, D. Harutyunyan and E. Novák, "Disentangling the ${ }^{16} \mathrm{O}$ cross section using light water and heavy water benchmark assemblies", Applied Radiation and Isotopes 133, pp. 45-50 (2018).

11. A. Kolros, O. Huml, M. Kř́ž and J. Kos, "Equipment for neutron measurements at VR-1 sparrow training reactor", Appl. Radiat. Isot. 68, pp. 570-574 (2010).

12. M. Koštál, F. Cvachovec, B. Jánský et al., "Neutron deep penetration through reactor pressure vessel and biological concrete shield of VVER-1000 Mock-Up in LR-0 reactor", Applied Radiation and Isotopes 94, pp. 672-683 (2016).

13. B. Ošmera, S. Zaritsky, VVER-1000 Benchmark Experiment in the LR-0 Reactor, Mock-up Description and Experimental Data, PROJECT REDOS FIKS-CT-200100120, Working Package No. 1, (2001).

14. M. Veškrna et al, "Digitalized two parametric system for gamma/neutron spectrometry", 18th Topical Meeting of the Radiation Protection \& Shielding Division of ANS, Knoxville, TN USA, (2014).

15. J. Cvachovec and F. Cvachovec, "Maximum likelihood estimation of a neutron spectrum and associated uncertainties", Advances in Military Technology, Vol. 1, 2, pp. $67-79$, (2008).

16. M. Košt'ál, J. Šoltés, L. Viererbl et al, "Measurement of neutron spectra in a silicon filtered neutron beam using stilbene detectors at the LVR-15 research reactor", Appl. Rad. and Isot., Vol. 128, pp. 41-48, (2017).

17. N. Otuka, E. Dupont, V. Semkova et al., Towards a more complete and accurate Experimental Nuclear Reaction Data Library (EXFOR): International collaboration between Nuclear Reaction Data Centres (NRDC), Nuclear Data Sheets 120 (2014) 272. 